\title{
More Smoke More Energy- Air-O-G: Energy from the Pollutant
}

\author{
Akhila $\mathrm{V} \mathrm{H}^{1}$, Anila $\mathrm{V} \mathrm{H}^{2}$ \\ ${ }^{I}$ Dept. of Computer Science and Engineering Government Engineering College, Sreekrishnapuram Palakkad, \\ Kerala, India \\ ${ }^{2}$ Dept. OF Electronics and Communication N.S.S College of Engineering Palakkad, Kerala, India
}

\begin{abstract}
India is home to four of the five cities in the world with the worst air pollution. With an annual average PM 2.5 level of $122 \mu \mathrm{g} / \mathrm{m}^{3}$, Delhi's air is the worst among global megacities with dense populations, as IndiaSpend reported in October 2016 based on WHO data. WHO experts acknowledge India faces a "huge challenge". Common causes of air pollution include too many cars, especially diesel-fueled vehicles, the heating and cooling of big buildings, waste management, agriculture and the use of coal or diesel generators for power. In this paper we propose an idea to reduce the emission of hazardous exhausts from the vehicles and large factories, were we include a mechanism to absorb the out coming hazardous exhausts within the system such that these doesn't enter into the earth's environment. The resulting non-hazardous exhaust from our system can be intelligibly utilized to generate electricity for the self-consumption purpose.
\end{abstract}

Index Terms: Green energy, Molecular Sieve Technology, Seebeck Effect (key words)

\section{Introduction}

The Air Quality Index study in the world states that India has a dangerously moved from " very unhealthy" air quality to "hazardous". The real time Air pollution study map shows the scenario[fig 1]. On October 30 and 31, 2016, from $9 \mathrm{pm}$ to the wee hours of the next morning, North India-especially parts of Delhi, Uttar Pradesh and Bihar-recorded PM 2.5 levels of over $500 \mu \mathrm{g} / \mathrm{m}^{3}$-exhibiting "beyond scale" pollution values, according to the database run by Berkeley Earth, an independent US research organization. Represented through heat maps, the database typically records the world's worst air quality-at the top of the scale, most between "very unhealthy" to "hazardous"-in dust-blown Xinjiang province in northwest China and parts of North India[1]. Our country is world's seventh most environmentally unsafe country [2]. In 2010, India's Central Pollution Control Board had presented the report according to which 180 cities in India

had particulate matter six times more than the permissible limit set by the World Health Organization in the air. Vehicles, biomass burning, fuel adulteration are few of the major causes of air pollution in India. As per the scientific studies it has been found out that traffic at low speed especially during congestion burn fuel ineffectually and emit 4 to 8 times more air pollutants. There are more than 40 different types of dangerous pollutants in the engine exhaust (diesel and gas). $70 \%$ of the air pollution is caused by vehicles. $30 \%$ children in Bangalore suffer from Asthma due to air pollution. The city is also regarded as the asthma capital of India. In NDTV it has been shown that Delhi is the most polluted city in the world and it left behind Beijing in air pollution. Industrial and vehicular emissions have caused the pollution to rise at this level in Delhi. Each day 1,400 new vehicles are added to Delhi roads.

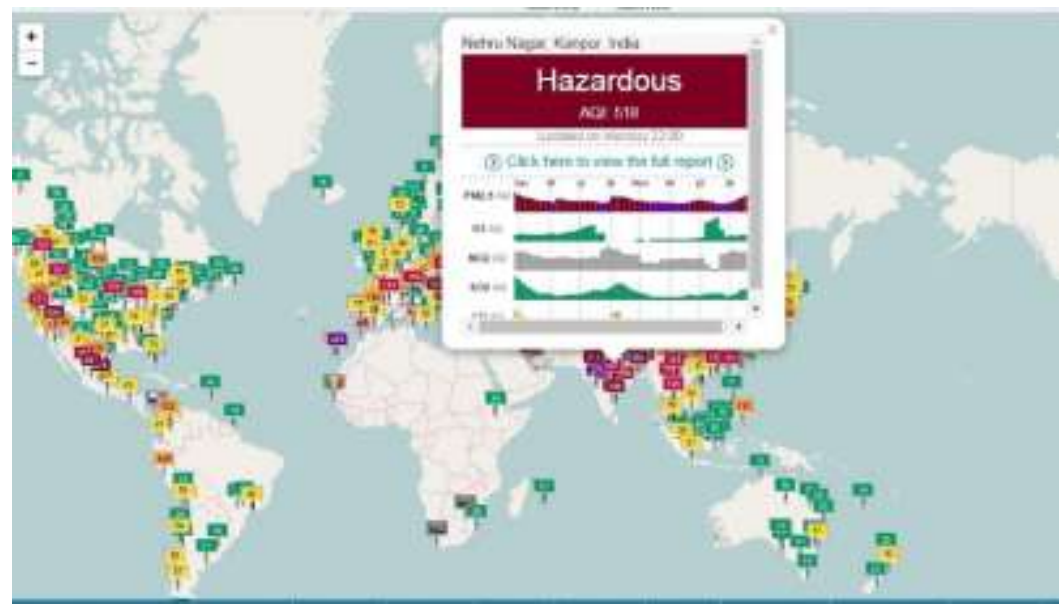

Fig 1: Air Quality Index (AQI) [Source: http://aqicn.org/map/world/ ] 


\section{Principles Used}

This paper uses the very basic principle of "Conservation Of Energy" with the core motive to increase the geological energy level or green energy level. Here, "Green energy" states the improved Air Quality which is healthy for the existence of the species on Mother Earth.

The paper aims to reduce pollutant level mainly due to vehicular emissions. We know from the first law of thermodynamics that, energy is neither created nor destroyed, it can just be transferred from one form to another. Thus, the exhaust vehicular emission can be converted to useful form of energy source, without releasing the hazardous exhausts into the air column. The smoke and heat produced from the stroke engine can be converted into useful form of energy source. For this we have analyzed the problem in two phases. In first phase, we have formulated a mechanism to draw energy from the exhaust and in the second, the exhaust filtration is done to remove harmful pollutants.

\section{Phase 1 : Energy Production From Exhaust}

We specifically analyze the exhaust air from the vehicles as a Column of fluid and apply the fluid dynamics. The exhaust before getting expelled into air has to be utilized for electricity production. The exhaust can be viewed as dual energy form i.e., heat and air (exhaust smoke). The heat energy can be absorbed and converted to electricity by "Thermoelectric Generator" and the exhaust smoke can be accelerated to produce electricity. For acceleration, exhaust gas has to pass through a mechanical energy converter (a generator or alternator). To rotate the rotor, the exhaust has to dash through the converter at a higher velocity. The idea of continuity equation is being used here for this. The main task in fluid dynamics is to find the velocity field describing the flow in a given domain. These encode the familiar laws of mechanics:

- Conservation of mass

- Conservation of momentum

\section{A. Conservation of mass}

\section{Continuity Equation}

When a fluid is in motion, it must move in such a way that mass is conserved. To see how mass conservation places restrictions on the velocity field, consider the steady flow of fluid through a duct (that is, the inlet and outlet flows do not vary with time). The inflow and outflow are one-dimensional, so that the velocity $\mathrm{V}$ and density trho are constant over the area A.

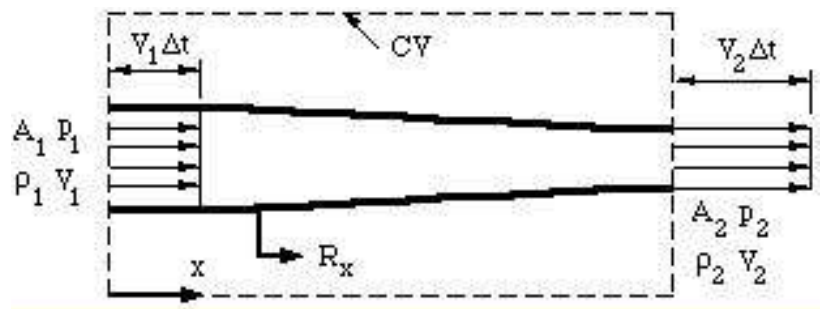

Fig 2: One-dimensional duct showing control volume.

Now we apply the principle of mass conservation. Since there is no flow through the side walls of the duct, what mass comes

in over A_1 goes out of A_2, (the flow is steady so that there is no mass accumulation). Over a short time interval $\backslash$ Delta $t$,

Volume flow in over $A_{1}=A_{1} V_{1} \Delta t$

Volume flow in over $A_{2}=A_{2} V_{2} \Delta t$

Therefore,

Mass in over $A_{1}=\rho A_{1} V_{1} \Delta t$

Mass in over $A_{2}=\rho A_{2} V_{2} \Delta t$

So,

$$
\rho \mathrm{A}_{1} \mathrm{~V}_{1}=\rho \mathrm{A}_{2} \mathrm{~V}_{2}
$$

This is a statement of the principle of mass conservation for a

steady, one-dimensional flow, with one inlet and one outlet. This equation is called the continuity equation for steady one-dimensional flow. For a steady flow through a control volume with many inlets and outlets, the net mass flow must be zero, where inflows are negative and outflows are positive.

So, we get the continuity equation:

$$
\partial \rho / \partial \mathrm{t}+\nabla \cdot(\rho \mathrm{v})=0 .
$$




\section{Incompressible continuity equation}

If the fluid is incompressible, $\rho=$ constant, independent of space and time, so that $\mathrm{D} \rho / \mathrm{Dt}=0$.

The continuity equation then reduces to:

$$
\nabla \cdot \mathrm{v}=0
$$

Which in Cartesian coordinates is

$$
\partial \mathrm{u} / \partial \mathrm{x}+\partial \mathrm{v} / \partial \mathrm{y}+\partial \mathrm{w} / \partial \mathrm{z}=0 \text {. }
$$

\section{B. Conservation of Momentum}

$\rho(\mathrm{u} \partial \mathrm{u} / \partial \mathrm{x}+\mathrm{v} \partial \mathrm{u} / \partial \mathrm{y})=-\partial \mathrm{P} / \partial \mathrm{x}+\mu\left(\partial^{2} \mathrm{u} / \partial \mathrm{x}^{2}+\partial^{2} \mathrm{u} / \partial \mathrm{y}^{2}\right)$

$\rho(\mathrm{u} \partial \mathrm{v} / \partial \mathrm{x}+\mathrm{v} \partial \mathrm{v} / \partial \mathrm{y})=-\partial \mathrm{P} / \partial \mathrm{y}+\mu\left(\partial^{2} \mathrm{v} / \partial \mathrm{x}^{2}+\partial^{2} \mathrm{v} / \partial \mathrm{y}^{2}\right)$

where $\mathrm{v}=(\mathrm{u}, \mathrm{v})$ is the velocity field. The first term on the RHS in Eqns. 4 and 5 refers to pressure forces, $\nabla \cdot \mathrm{P}$. The rest of the RHS describes viscous forces, $\mu \nabla^{2} \mathrm{v}$. The LHS is the momentum change that any element experiences as it moves between regions of different velocity in the flow field. This has the dimensions of a force, and is referred to as the inertia force, $\rho \mathrm{v} \cdot \nabla \mathrm{v}$.

\section{Phase 2: Exhaust Filtration}

The analysis by researchers at the University of East Anglia and the Global Carbon Project, a group of scientists who measure how much carbon dioxide humans emit every year and how much is subsequently absorbed by plants, land surfaces and oceans, showed emissions growth remained below one per cent despite GDP growth exceeding three per cent.The Global Carbon Budget analysis showed that, in spite of a lack of growth in emissions, the growth in atmospheric CO2 concentration was a record-high in 2015, and could be a record again in 2016 due to weak carbon sinks. "Part of the CO2 emissions are absorbed by the ocean and by trees. With temperatures soaring in 2015 and 2016, less CO2 was absorbed by trees because of the hot and dry conditions related to the El Nino event. Atmospheric CO2 levels have exceeded 400 parts per million (ppm) and will continue to rise and cause the planet to warm until emissions are cut down to near zero."

The 2016 edition of BP's authoritative Statistical Review of World Energy offers a lot of startling statistics and revelations. According to the report, India's energy consumption rose by 5.2\% in 2015. [3], For comparison's sake, the US saw a decrease of just under $13 \%$ and China decreased by $1.5 \%$. The additional CO2 India added to the atmosphere in 2014 (157MT) was greater than that added by the US in 2013 (155.T). The American economy is ten times as big as India's and consumes nearly four times as much energy. The largest increase in global CO2 emissions - 5.3\% - came from India, making it the world's fastest-growing major polluter. This alarming share of $\mathrm{CO} 2$ emission by India is contributed by the vehicular emission and industries. Thus as per reports, our major task will be cut down the $\mathrm{CO} 2$ emission to near zero. The Molecular sieve technology can be used here. A molecular sieve is a material with pores (very small holes) of uniform size. These pore diameters are similar in size to small molecules, and thus large molecules cannot enter or be absorbed, while smaller molecules can. As a mixture of molecules migrate through the stationary bed of porous, semi-solid substance referred to as a the sieve (or matrix), the components of highest molecular weight (which are unable to pass into the molecular pores) leave the bed first, followed by successively smaller molecules. The diameter of a molecular sieve is measured in angstroms $(\AA)$ or nanometers (nm).

Materials can be classified into Microporous, Mesoporous and Macroporous. According to IUPAC notation, microporous materials have pore diameters of less than $2 \mathrm{~nm}(20 \AA)$ and macroporous materials have pore diameters of greater than $50 \mathrm{~nm}(500 \AA)$; the mesoporous category thus lies in the middle with pore diameters between 2 and $50 \mathrm{~nm}(20-500 \AA)$. $5 \AA$ molecular sieves are often utilized in the petroleum industry, especially for the purification of gas streams and in the chemistry laboratory for separating compounds and drying reaction starting materials. They contain tiny pores of a precise and uniform size, and are mainly used as an adsorbent for gases and liquids.

Thus Microporous sieve can be used for $\mathrm{CO} 2$ absorbtion. One of the Microporous Molecular sieve is Zeolite. Zeolites are used to adsorb a variety of materials. This includes applications in drying, purification, and separation. They can remove water to very low partial pressures and are very effective desiccants, with a capacity of up to more than $25 \%$ of their weight in water. They can remove volatile organic chemicals from air streams, separate isomers and mixtures of gases. A widely used property of zeolites is that of gas separation.
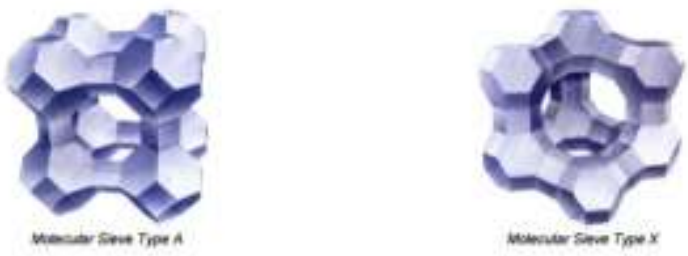

Fig 3 
[4], These crystals are characterized by a three-dimensional pore system, with pores of precisely defined diameter. The corresponding crystallographic structure is formed by tetrahedras of (AlO4) and ( $\mathrm{SiO} 4)$. These tetrahedras are the basic building blocks for various zeolite structures, such as zeolites A and X, the most common commercial adsorbents(fig 3). Due to the presence of alumina, zeolites exhibit a negatively charged framework, which is counter-balanced by positive cations resulting in a strong electrostatic field on the internal surface. These cations can be exchanged to fine-tune the pore size or the adsorption characteristics.
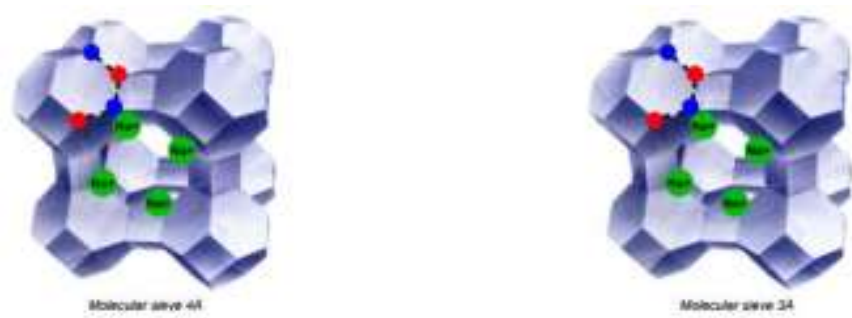

Fig 4: $3 \mathrm{~A}$ and $4 \mathrm{~A}$

For instance, the sodium form of zeolite A has a pore opening of approximately 4 Ångstrom (4 x 10-10 $\mathrm{m})$, called $4 \mathrm{~A}$ molecular sieve. If the sodium ion is exchanged with the larger potassium ion, the pore opening is reduced to approximately 3 Ångstrom (3A molecular sieve) fig 4 . On ion exchange with calcium, one calcium ion replaces two sodium ions. Thus, the pore opening increases to approximately $5 \AA$ Angstrom (5A molecular sieve). Ion exchange with other cations is sometimes used for particular separation purposes. The pore opening of the sodium form of zeolite $\mathrm{X}(13 \mathrm{X})$ is approximately 8 Angstrom.

From [5], Zeolite 5A and 13X can be used for $\mathrm{CO} 2$ absorption. 5A has Composition,

$0.80 \mathrm{CaO}: 0.20 \mathrm{Na} 2 \mathrm{O}: 1 \mathrm{Al} 2 \mathrm{O} 3: 2.0 \pm 0.1 \mathrm{SiO} 2$ : x H2O

Divalent calcium ions in place of sodium cations give apertures of $\sim 5 \AA$ which exclude molecules of effective diameter $>5 \AA$ A e.g., all 4-carbon rings, and iso-compounds. $13 \mathrm{X}$ has Composition,

$1 \mathrm{Na} 2 \mathrm{O}: 1 \mathrm{Al} 2 \mathrm{O} 3: 2.8 \pm 0.2 \mathrm{SiO} 2: \mathrm{xH} 2 \mathrm{O}$

The sodium form represents the basic structure of the type X family, with an effective pore opening in the $9101 / 4$ range. Major application of $13 \mathrm{X}$ is Commercial gas drying, air plantfeed purification (simultaneous $\mathrm{H} 2 \mathrm{O}$ and $\mathrm{CO} 2$ removal) and liquid hydrocarbon/natural gas sweetening (H2S and mercaptan removal).

\section{System Architecture}

The Air-O-G system architecture contains two phases as discussed above. In the first phase, energy is converted to electricity. The components in phase 1 are:

a) Stroke engine

b) Higher Diameter Exhaust Pipe

c) Seebeck Generator

d) Rotor

e) Generator

The Second Phase is for the absorption of harmful pollutants, mainly CO2. The major components in phase 2 is : carbon dioxide removal assembly (CDRA), consisting of two layers of Zeolite. A zeolite 13X connected to a zeolite $5 \mathrm{~A}$ layer. As the air passes through the zeolite $13 \mathrm{X}$ bed, water gets trapped and removed from the air. The dried air goes into the zeolite $5 \mathrm{~A}$ bed where carbon dioxide gets trapped and removed. The outgoing air is then dry and free from carbon dioxide. The fig 5 gives the system architecture of Air-O-G.

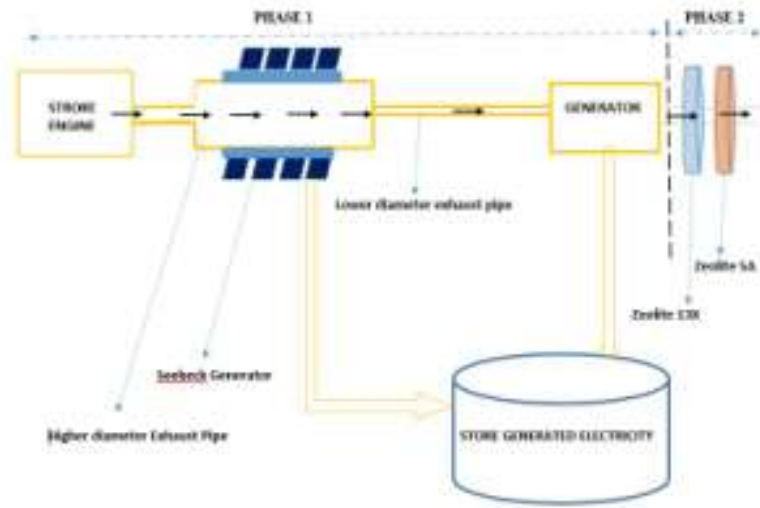

Fig 5: System Architecture of Air-O-G 
In Air-O-G, the hazardous exhaust from the stroke engine is made to pass through a higher diameter exhaust pipe. While passing through this pipe, the exhaust will be having a higher temperature. The heat liberated from this exhaust can be converted to electricity by "Seebeck Generator". This produced energy is being stored up for future use.

A thermoelectric generator (TEG), also called a Seebeck generator, is a solid state device that converts heat (temperature differences) directly into electrical energy through a phenomenon called the Seebeck effect (a form of thermoelectric effect). Thermoelectric generators function like heat engines, but are less bulky and have no moving parts.

Thermoelectric materials generate power directly from heat by converting temperature differences into electric voltage. These materials must have both high electrical conductivity $(\sigma)$ and low thermal conductivity ( $\kappa)$ to be good thermoelectric materials. Having low thermal conductivity ensures that when one side is made hot, the other side stays cold, which helps to generate a large voltage while in a temperature gradient. The measure of the magnitude of electrons flow in response to a temperature difference across that material is given by the Seebeck coefficient (S).

The exhaust is then made to pass through a lower diameter tube ( a narrow tube), which makes the air to rush in a comparatively higher velocity towards the Rotor. The rotation of the rotor blades helps in the generation of the Electricity via an attached Generator to this rotor. The Electricity produced to stored up for future use. In case of vehicles, this produced electricity can be used up for lighting the headlight and indicators or these can be used to charge the battery as well.

The air after exiting the phase 1 is made to pass through two zeolite beds, which constitutes the phase 2 of Air-O-G. This phase 2 contains two Zeolite Beds. Zeolite $13 \mathrm{x}$ followed by $5 \mathrm{~A}$. These helps to absorb the harmful pollutants and also helps to absorb $\mathrm{CO} 2$ in specific and this completely reduces the $\mathrm{CO} 2$ emission into the atmosphere.

\section{Conclusion}

Air-O-G introduces an architecture for reducing the $\mathrm{CO} 2$ emission and to convert the heat and exhaust air from the vehicular emission to useful energy. Thus, Air-O-G helps for converting wasted heat and momentum to useful form of energy.

\section{Acknowledgment}

This research was supported by faculty of Government Engineering College, Barton Hill, Trivandrum and N.S.S College Of Engineering, Palakkad. We are thankful to our friends and family who provided expertise that greatly assisted the research, although they may not agree with all of the interpretations provided in this paper.

\section{References}

[1]. http://www.hindustantimes.com/india-news/india-s-air-quality-among-world-s-worst-over-diwali-weekendreport/story8NIJIXGlzSAusoHj867upL.html

[2]. http://www.mapsofindia.com/my-india/india/what-kind-of-air-are-we-breathing-air-pollution-in-india

[3]. http://www.earthday.org/2016/06/14/largest-increase-global-co2-emissions-energy-use-2015-came-india/ http://www.gracedavison.com/eusilica/Adsorbents/product/zeolite

[4]. http://www.sigmaaldrich.com/chemistry/chemical- synthesis/learning-center/technical-bulletins/al- 1430/molecular-sieves.html

[5]. https://en.wikipedia.org/wiki/Thermoelectric_generator
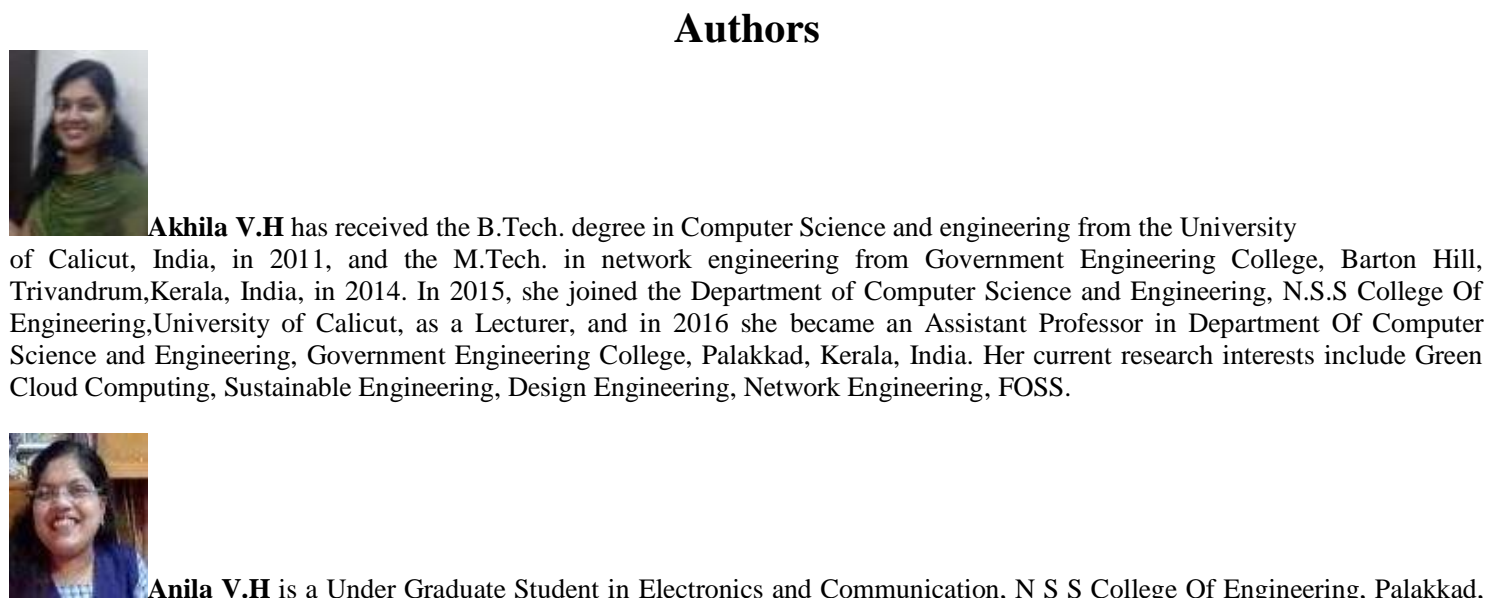
Kerala (Under Kerala Technological University). Her area of interests includes Mechanical Engineering, Aerospace Engineering, Sustainable Engineering and Design Engineering. 